\title{
Antenna design for fast ion collective Thomson scattering diagnostic for the international thermonuclear experimental reactor
}

Leipold, Frank; Furtula, Vedran; Salewski, Mirko; Bindslev, Henrik; Korsholm, Søren Bang; Meo, Fernando; Michelsen, Poul; Moseev, Dmitry; Nielsen, Stefan Kragh; Stejner Pedersen, Morten

Published in:

Review of Scientific Instruments

Link to article, DOI:

$10.1063 / 1.3212567$

Publication date:

2009

Document Version

Publisher's PDF, also known as Version of record

Link back to DTU Orbit

Citation (APA):

Leipold, F., Furtula, V., Salewski, M., Bindslev, H., Korsholm, S. B., Meo, F., Michelsen, P., Moseev, D., Nielsen, S. K., \& Stejner Pedersen, M. (2009). Antenna design for fast ion collective Thomson scattering diagnostic for the international thermonuclear experimental reactor. Review of Scientific Instruments, 80(9), 093501. https://doi.org/10.1063/1.3212567

\section{General rights}

Copyright and moral rights for the publications made accessible in the public portal are retained by the authors and/or other copyright owners and it is a condition of accessing publications that users recognise and abide by the legal requirements associated with these rights.

- Users may download and print one copy of any publication from the public portal for the purpose of private study or research.

- You may not further distribute the material or use it for any profit-making activity or commercial gain

- You may freely distribute the URL identifying the publication in the public portal 


\title{
Antenna design for fast ion collective Thomson scattering diagnostic for the international thermonuclear experimental reactor
}

\author{
F. Leipold, ${ }^{\text {a) }}$ V. Furtula, M. Salewski, H. Bindslev, S. B. Korsholm, F. Meo, \\ P. K. Michelsen, D. Moseev, S. K. Nielsen, and M. Stejner \\ Association Euratom-Ris $\phi$ National Laboratory for Sustainable Energy, Technical University of Denmark, \\ DK-4000 Roskilde, Denmark
}

(Received 29 May 2009; accepted 1 August 2009; published online 11 September 2009)

\begin{abstract}
Fast ion physics will play an important role for the international thermonuclear experimental reactor (ITER), where confined alpha particles will affect and be affected by plasma dynamics and thereby have impacts on the overall confinement. A fast ion collective Thomson scattering (CTS) diagnostic using gyrotrons operated at $60 \mathrm{GHz}$ will meet the requirements for spatially and temporally resolved measurements of the velocity distributions of confined fast alphas in ITER by evaluating the scattered radiation (CTS signal). While a receiver antenna on the low field side of the tokamak, resolving near perpendicular (to the magnetic field) velocity components, has been enabled, an additional antenna on the high field side (HFS) would enable measurements of near parallel (to the magnetic field) velocity components. A compact design solution for the proposed mirror system on the HFS is presented. The HFS CTS antenna is located behind the blankets and views the plasma through the gap between two blanket modules. The viewing gap has been modified to dimensions $30 \times 500 \mathrm{~mm}^{2}$ to optimize the CTS signal. A 1:1 mock-up of the HFS mirror system was built. Measurements of the beam characteristics for millimeter-waves at $60 \mathrm{GHz}$ used in the mock-up agree well with the modeling. () 2009 American Institute of Physics. [doi:10.1063/1.3212567]
\end{abstract}

\section{INTRODUCTION}

Plasmas for thermonuclear fusion contain a highly nonthermal population of fast ions which may carry about one third of the plasma kinetic energy. Ions are accelerated to high energies by neutral beam injection (NBI) or ion cyclotron resonance heating (ICRH). They are also born in the deuterium-tritium fusion reaction which is the workhorse of particle heating for reactors which approach or reach the break-even condition. The dynamics of the fast ions plays an increasingly central role as fusion power plant conditions are approached: fast ions must be confined in the plasma long enough to heat the bulk plasma. However, they also drive instabilities and may leave the plasma before they have transferred their excess energy to the bulk plasma. It is therefore essential to understand the dynamics of fast ions in fusion plasmas. ${ }^{1-3}$ Collective Thomson scattering (CTS) offers the opportunity to diagnose confined fast ions resolved in space, velocity space, and in time. In CTS, one monitors a signal of radiation resulting from scattering of a powerful beam of probe radiation on collective fluctuations in the electron distribution. The velocity distribution along a chosen direction (given by the scattering geometry) can be inferred from the spectral content of the scattered radiation. ${ }^{4-6}$ As the fast ion distribution may be highly anisotropic, the velocity distribution should optimally be resolved in at least two directions, where perpendicular and parallel to the magnetic field is a good option. The spatial location of the measuring volume and the spatial resolution are given by the intersec-

\footnotetext{
${ }^{a)}$ Electronic mail: leip@ risoe.dtu.dk.
}

tion of the probe beam and the receiver beam collecting the scattered signal. The CTS diagnostic has been previously successfully applied in the JET experiment ${ }^{7}$ and in the TEXTOR experiment. ${ }^{8}$ Preliminary results of the CTS system at ASDEX Upgrade have been presented. ${ }^{9}$ Here we discuss the CTS system for the international thermonuclear experimental reactor (ITER) which is designed to meet ITER diagnostics requirements. ${ }^{10-12}$ The CTS system on ITER is composed of two subsystems: the near perpendicular velocity components of the fast ions with respect to the magnetic field can be resolved with a receiver antenna on the low field side (LFS), whereas a receiver antenna on the high field side (HFS) will be required for resolution of the near parallel velocity components. It has been shown that the CTS signal to be measured in a burning ITER plasma will be dominated by alpha particles whereas contributions from NBI and ICRH will be relatively small. ${ }^{13-15}$ Space for the CTS subsystem with the antenna on the LFS has been reserved, but the other subsystem with the antenna on the HFS is not part of the ITER baseline design. ${ }^{16}$ In this paper, we present a compact design solution for a CTS antenna on the HFS. In Sec. II, we briefly describe the CTS system at ITER. Section III is focused on the HFS antenna. In Sec. IV, measurements and simulations of beam propagation through a 1:1 mock-up of the envisaged HFS ITER CTS system are presented and conclusions are drawn in Sec. V.

\section{THE CTS DIAGNOSTIC FOR ITER}

Following the ITER design review, the CTS subsystem with the receiver antenna on the LFS has been enabled in the current ITER baseline design. ${ }^{16}$ With this CTS subsystem, 


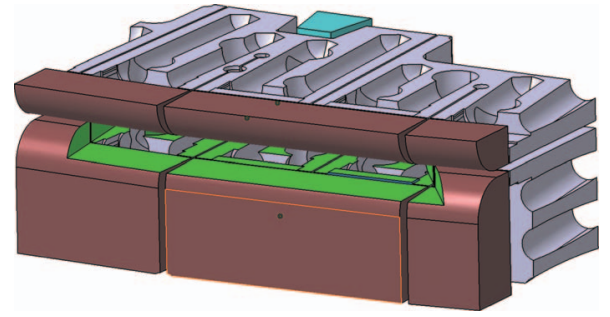

FIG. 1. (Color) The additional envisaged CTS system on ITER with an antenna on the HFS would require a slit (green, $500 \times 30 \mathrm{~mm}^{2}$ ) in the blanket of the inner wall.

the near perpendicular velocities of fast ions can be measured at several locations simultaneously. Here, we discuss a CTS subsystem with the receiver antenna on the HFS which would enable resolution of near parallel velocities of the fast ions. The design of both CTS subsystems has progressed considerably. ${ }^{11,17-19}$ The envisaged additional CTS system with the receiver on the HFS will utilize a $60 \mathrm{GHz}$ gyrotron with $1 \mathrm{MW}$ power as probing beam. The system is capable of resolving the fast ion distribution in at least 16 velocity intervals with a spatial resolution of at least $1 / 10$ th of the minor radius and a time resolution better than $100 \mathrm{~ms} .{ }^{10}$ The gyrotron radiation enters the plasma from the LFS equatorial port plug and is received on the HFS. In terms of technology, the CTS system for ITER differs from the previous successful CTS experiments on other machines due to much larger heat and neutron fluxes in the ITER experiment. This radiative environment results in different choices in materials and design as compared to other machines. Second, the CTS system for ITER has to be designed within the geometric constraints given by the machine. To protect the other systems from the severe neutron and heat flux (e.g., the superconducting coils), the slits providing optical access to the plasma have to be as narrow as possible. The scattered CTS signal captured on the HFS in the vessel first needs to go from the plasma through the slit in the blanket of the inner wall of the vessel. Therefore, a slit with a dimension of $500 \mathrm{~mm}$ width and $30 \mathrm{~mm}$ height should be cut in the blanket. Figure 1 shows the part of the blanket out of which the slit needs to be cut. A microwave beam passing through this aperture can be described as an anisotropic Gaussian beam for which the horizontal beam width is $100 \mathrm{~mm}$ and the vertical beam width is $10 \mathrm{~mm}$. The aperture should be large enough to capture 1.6 times the Gaussian beam width in order to catch $99.9 \%$ of the radiation and to avoid reflections. This will result in an aperture opening of $300 \times 30 \mathrm{~mm}^{2}$. The minimum required vertical slit dimension was obtained from a full wave simulation. The beam passing this aperture is anisotropic. However, at the receiver antenna, an isotropic beam is preferred because of the design of the receiver horn antenna. Therefore, mirrors behind the blanket are required to reshape the anisotropic Gaussian beam into an isotropic Gaussian beam. We refer to this beam as the center beam. The mirror system and the horn antenna are designed for optimal transmission of the center beam. Since the fast ion distribution shall be measured at several locations in the plasma, beams from different incident angles $\left( \pm 15^{\circ}\right)$ in the horizontal plane shall be accepted. This requires the enlarged

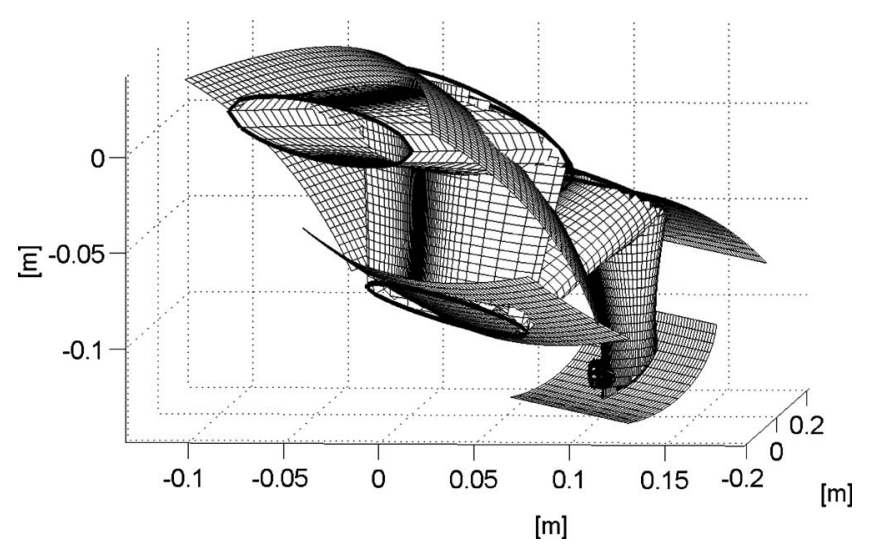

FIG. 2. Calculated mirror shapes and transmission of the anisotropic Gaussian beam. The four mirrors are shown as toroidal surfaces. The beam is indicated by the surface containing $99.9 \%$ of the beam power. The beam enters the receiver at the top and leaves at the bottom.

aperture in the horizontal direction with a width of approximately $500 \mathrm{~mm}$. Beams coming from different angles with respect to the center beam require different horn positions since the mirrors are fixed. The distance between these horn antennas determine the distance between measurement locations in the plasma. In this work, only the center beam is considered.

\section{DESIGN OF THE HFS ANTENNA}

The space for the mirrors behind the blanket is limited to $190 \mathrm{~mm}$ vertically, $300 \mathrm{~mm}$ horizontally, and $230 \mathrm{~mm}$ radially. Keeping these boundary conditions in mind, the mirror shapes for a quasioptical transmission line were computed by calculating the propagation of an anisotropic Gaussian beam. The beam parameters at the slit in the blanket are given by the slit dimensions. The distance between two adjacent measurement locations in the plasma is determined by the distance between two adjacent horn antennas and therefore limited by the beam size. A small beam size allows a close arrangement of the horn antennas and therefore allows decreasing the distance between measurement locations in the plasma. In order to reshape an anisotropic beam with given widths and curvatures at the beginning and at the end of the beam, at least two mirrors are required. Due to the large difference in the beam dimensions between slit and horn antenna, the reshaping should be performed on a propagation path long enough to be well within the paraxial approximation of Gaussian beam propagation. Due to the limited space, a long, straight path is not possible. Therefore, two additional mirrors were employed to increase the optical path length between the slit and the horn antenna. The mirror shapes, which are required to reshape the beam according to these boundary conditions, are calculated. Mathematically, the mirrors are surfaces of tori. The mirror surfaces and the beam shape are shown in Fig. 2. The beam enters the receiver at the top and leaves at the bottom. Figure 3 shows the beam width for the two major axes along the path of propagation through the quasioptical transmission line. The triangles indicate positions of the mirrors, the diamond indicates the position of the beam waist. At this position, the isotropic Gaussian beam has a waist of $4.5 \mathrm{~mm}$, and it is 


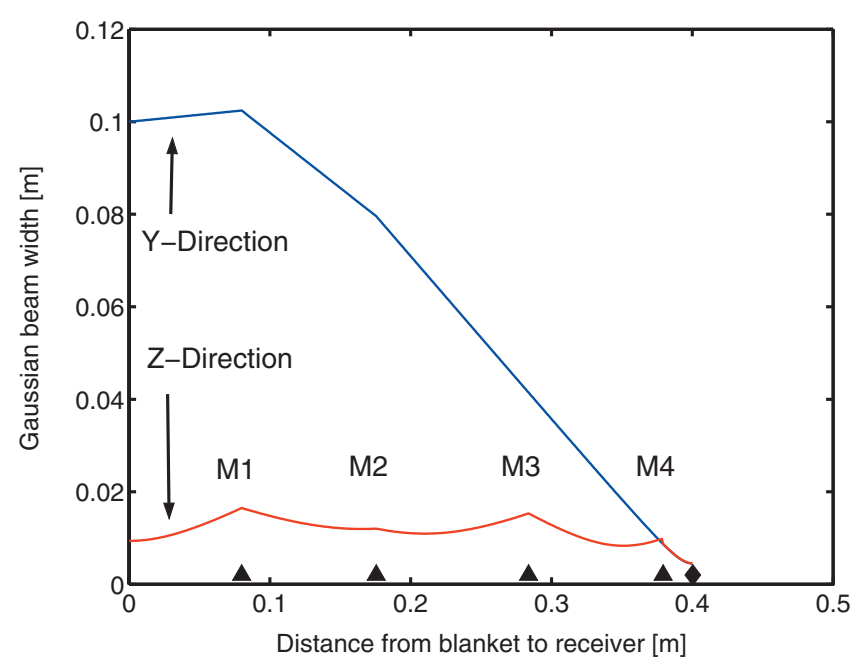

FIG. 3. (Color) Gaussian beam widths in the two orthogonal directions in the transmission lines. Between the reference point and mirror M1, the two orthogonal directions coincide with the $y$-axis (blue curve) and the $z$-axis (red curve). The triangles indicate the mirror positions M1 to M4 and the diamond indicates the position of the waist which is located slightly inside the horn antenna. See also Fig. 7.

located $3.4 \mathrm{~mm}$ inside the horn antenna. The horn antenna is connected to a fundamental waveguide by means of a taper. The data for the mirror surfaces were imported into the computer-aided design (CAD) software CATIA V5 where the hardware design was made. Figure 4 shows the mirror assembly, and Fig. 5 shows the mirror assembly integrated in the blanket. Figure 5 shows also the design which has been set up in the laboratory as a 1:1 mock-up in order to verify the calculation of the beam propagation. The corrugated horn antenna shall accept an isotropic Gaussian beam with a beam waist of $4.5 \mathrm{~mm}$ corresponding to a divergence angle of $20.3^{\circ}$. A corrugated horn antenna for a frequency of $60 \mathrm{GHz}$ was designed and built based on previous work. ${ }^{20}$

\section{MEASUREMENT OF THE BEAM CHARACTERISTIC OF THE HFS ANTENNA}

\section{A. The characteristic of the corrugated horn antenna}

In order to verify whether the horn meets the requirements, a $60 \mathrm{GHz}$ oscillator was mounted at the port of the

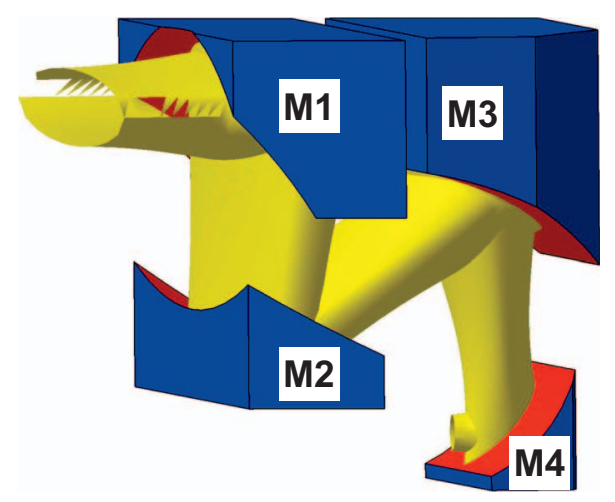

FIG. 4. (Color) Mirror assembly (mirrors M1-M4) for the HFS CTS transmission line. The red surfaces are the mirror surfaces calculated and imported into CATIA. The yellow surface represents 1.6 times the Gaussian beam width of the anisotropic Gaussian beam. See also Fig. 7.

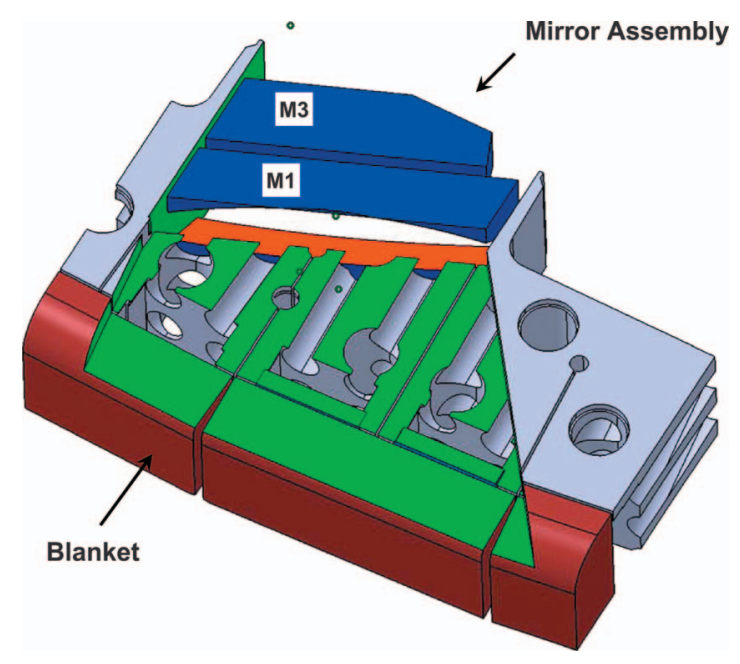

FIG. 5. (Color) Mirror assembly for the proposed antenna on the HFS integrated in the blanket of the inner wall of ITER. The cutting surface of the blanket is depicted in green. Only the mirrors M1 and M3 (blue) and the surface of mirror M2 (red) of the mirror assembly can be seen. See also Figs. 4 and 7.

horn, and the device was used as a millimeter-wave source. The beam pattern in front of the horn opening was measured at various distances from the horn. Figure 6 shows the Gaussian beam width versus the distance for two orthogonal directions. From these measurements, the divergence angle is obtained. The beam was found to be slightly elliptical with a measured deviation of less than $3 \%$ from the ideal circular pattern. The divergence angles for the major and minor axes were found to be $19.0^{\circ}$ and $19.8^{\circ}$, respectively. This corresponds to beam waists of 4.8 and $4.6 \mathrm{~mm}$ (inside the horn antenna), respectively. The measured beam sizes in two orthogonal directions are in good agreement with the designed beam sizes.

\section{B. The characteristic of the mirror system}

In order to test the propagation of radiation through the entire receiver optics, the horn antenna is integrated into the

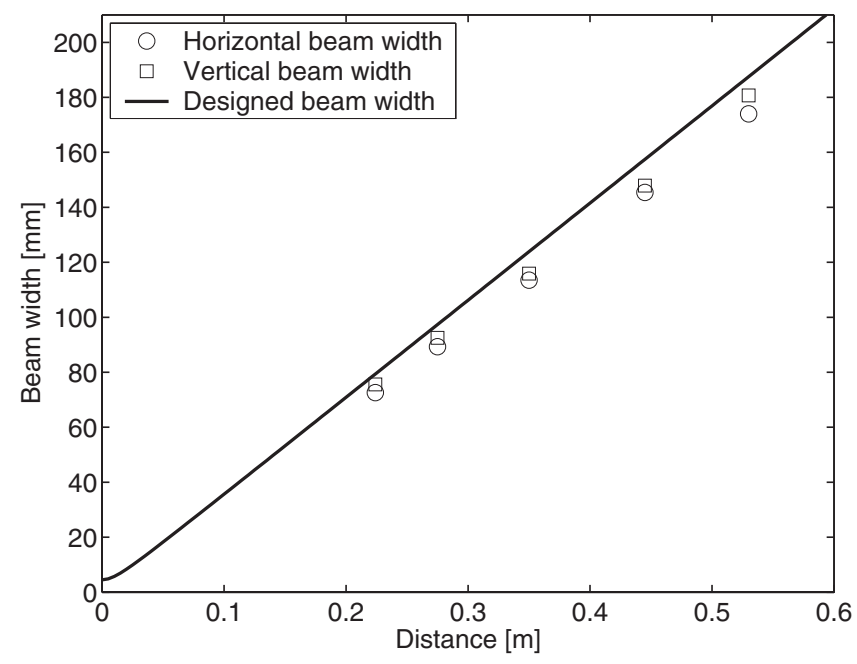

FIG. 6. Gaussian widths of the beam leaving the horn antenna. The horizontal and vertical beam widths should be identical, as the horn has a circular symmetry. The error is less than $3 \%$ between the measured beams in two orthogonal directions. The solid line represents the designed beam width. 


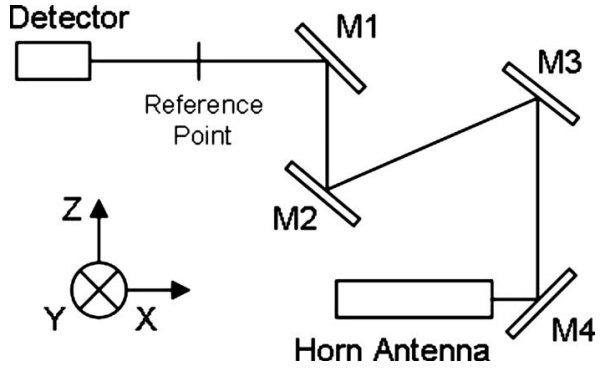

FIG. 7. Schematic of the mirrors (M1-M4), the horn antenna, and the detector diode. The origin of the graphs in Fig. 3 and Fig. 9 coincides with the reference point. The distance on the abscissa in Fig. 3 is measured from the reference point toward the horn antenna; in Fig. 9 it is measured toward the detector.

mirror assembly (see Fig. 7). A detector, which is movable in the $y$-z-plane, is used to characterize the beam pattern at various distances $x$ from the reference point (Fig. 7). Figure 8 shows a contour plot of the beam intensity in the $y$-z-plane at a distance of $166 \mathrm{~mm}$ from the reference point. The distance between the reference point and M1 is $80 \mathrm{~mm}$. The beam widths in the $y$ - and $z$-directions at various distances from the reference point are shown in Fig. 9. The circles in Fig. 9 show the divergence of the beam from the reference point in the $z$-direction. The beam waist is calculated from the divergence angle and is found to be $8.3 \mathrm{~mm}$. This is within an error of $13 \%$ compared to the calculated value of $9.4 \mathrm{~mm}$. The squares in Fig. 9 show the beam width at approximately $100 \mathrm{~mm}$ in the $y$-direction. A converging beam can be seen in the $y$-direction. The calculation (solid curve through the squares in Fig. 9) shows a converging beam with a beam waist of $47 \mathrm{~mm}$ at a distance $2.7 \mathrm{~m}$ away from the reference point. The beam could not be measured at distances larger than $0.35 \mathrm{~m}$ from the reference point due to a low signal-to-noise ratio which gives rise to a high uncertainty especially for the convergence angle. However, the characteristic is in agreement with the calculation. Microwave sources with higher power will be employed to overcome this problem.

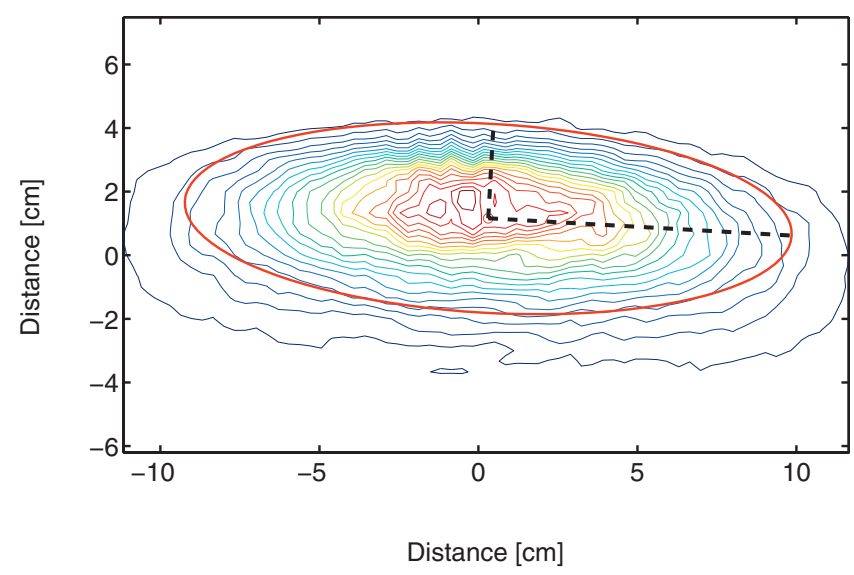

FIG. 8. (Color) Contour plot shows the radiation intensity in the $y$-z-plane at a distance of $166 \mathrm{~mm}$ from the reference point (see Fig. 7). The dashed lines show the width in the direction of the major axes of the anisotropic beam.

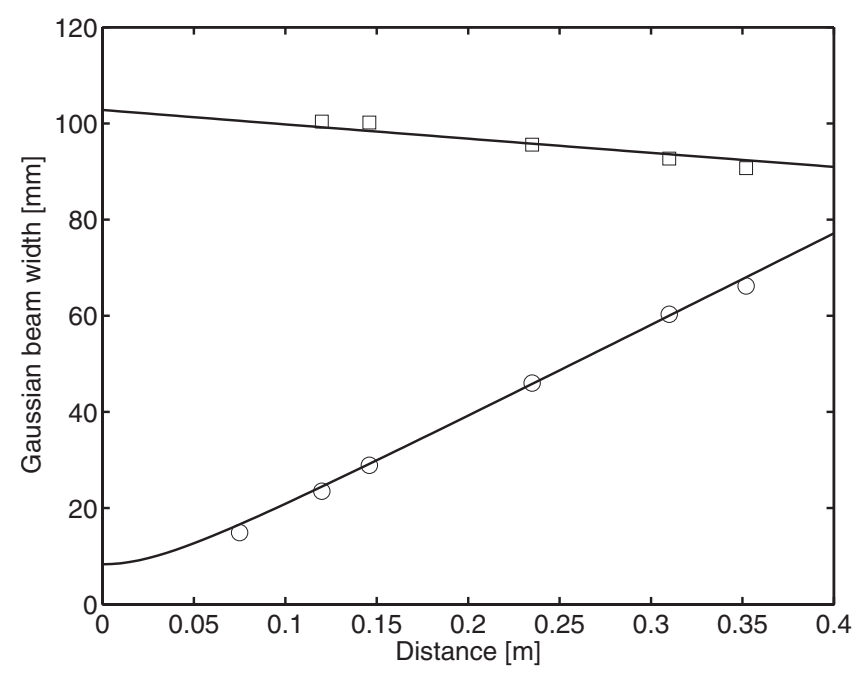

FIG. 9. Propagation of the $y$ - and $z$-component of the anisotropic Gaussian beam into the vessel. The squares show the horizontal component and the circles show the vertical component. The solid lines represent the calculated beam widths. See also Fig. 7.

\section{CONCLUSION}

A design for an additional antenna on the HFS for the CTS system at ITER was discussed. Such an antenna is necessary to resolve the near parallel velocity components of the fast ion distribution. Here we presented a compact design solution of an antenna for such a system which can be accommodated on the HFS of ITER. The required shape of the mirrors in order to ensure an acceptable beam has been computed. The antenna system, consisting of mirror assembly and horn antenna has been verified experimentally with a 1:1 mock-up.

\section{ACKNOWLEDGMENTS}

This work, supported by the European Communities under the contract of Association between EURATOM/Ris $\varnothing$ DTU, was partly carried out within the framework of the European Fusion Development Agreement. The views and opinions expressed herein do not necessarily reflect those of the European Commission.

${ }^{1}$ W. Heidbrink and G. Sadler, Nucl. Fusion 34, 535 (1994).

${ }^{2}$ ITER Physics Expert Group on Energetic Particles, Heating and Current Drive, Nucl. Fusion 39, 2471 (1999).

${ }^{3}$ A. Fasoli, C. Gormenzano, H. Berk, B. Breizman, S. Briguglio, D. Darrow, N. Gorelenkov, W. Heidbrink, A. Jaun, S. Konovalov, R. Nazikian, J.-M. Noterdaeme, S. Sharapov, K. Shinohara, D. Testa, K. Tobita, Y. Todo, G. Vlad, and F. Zonca, Nucl. Fusion 47, S264 (2007).

${ }^{4}$ H. Bindslev, Plasma Phys. Controlled Fusion 35, 1615 (1993).

${ }^{5}$ H. Bindslev, J. Atmos. Terr. Phys. 58, 983 (1996).

${ }^{6}$ H. Bindslev, Rev. Sci. Instrum. 70, 1093 (1999).

${ }^{7}$ H. Bindslev, J. A. Hoekzema, J. Egedal, J. A. Fessey, T. P. Hughes, and J. S. Machuzak, Phys. Rev. Lett. 83, 3206 (1999).

${ }^{8}$ H. Bindslev, S. K. Nielsen, L. Porte, J. A. Hoekzema, S. B. Korsholm, F. Meo, P. K. Michelsen, S. Michelsen, J. Oosterbeek, E. L. Tsakadze, E. Westerhof, P. Woskow, and the TEXTOR team, Phys. Rev. Lett. 97, 205005 (2006)

${ }^{9}$ F. Meo, H. Bindslev, S. B. Korsholm, V. Furtula, F. Leuterer, F. Leipold, P. K. Michelsen, S. K. Nielsen, M. Salewski, J. Stober, D. Wagner, and P. Woskov, Rev. Sci. Instrum. 79, 10E501 (2008).

${ }^{10}$ H. Bindslev, F. Meo, E. Tsakadze, S. B. Korsholm, and P. Woskov, Rev. Sci. Instrum. 75, 3598 (2004).

${ }^{11}$ F. Meo, H. Bindslev, S. Korsholm, E. Tsakadze, C. Walker, P. Woskov, 
and G. Vayakis, Rev. Sci. Instrum. 75, 3585 (2004).

${ }^{12}$ F. P. Orsitto, J. M. Noterdaeme, A. E. Costley, and A. J. H. Donne, Nucl. Fusion 47, 1311 (2007).

${ }^{13}$ M. Salewski, O. Asunta, L.-G. Eriksson, H. Bindslev, V. Hynönen, S. B. Korsholm, T. Kurki-Suonio, F. Leipold, F. Meo, P. K. Michelsen, S. K. Nielsen, and J. Roenby, Plasma Phys. Controlled Fusion 51, 035006 (2009).

${ }^{14}$ M. Salewski, L.-G. Eriksson, H. Bindslev, S. B. Korsholm, F. Leipold, F. Meo, P. K. Michelsen, and S. K. Nielsen, Nucl. Fusion 49, 025006 (2009).

${ }^{15}$ J. Egedal, H. Bindslev, R. Budney, and P. Woskov, Nucl. Fusion 45, 191 (2005).

${ }^{16}$ A. Costley, S. Allen, P. Andrew, L. Bertalot, R. Barnsley, X. Duan, A. Encheva, C. Ingesson, D. Johnson, H. Lee, Y. Kawano, A. Krasilnikov, V. Kumar, Y. Kusama, E. Marmar, S. Pak, C. Pitcher, C. Rao, G. Saibene, D.
Thomas, P. Thomas, P. Vasu, G. Vayakis, C. Walker, Q. Yang, V. Zaveriaev, and J. Zhao, Proceedings of the 22nd IAEA Conference (IAEA, Trieste/Vienna, 2008).

${ }^{17}$ E. Tsakadze, H. Bindslev, S. Korsholm, A. Larsen, F. Meo, P. Michelsen, S. Michelsen, A. Nielsen, S. Nimb, B. Lauritzen, E. Nonbøl, and N. Dubois, Fusion Sci. Technol. 53, 69 (2008).

${ }^{18}$ S. B. Korsholm, H. Bindslev, V. Furtula, F. Leipold, F. Meo, P. K. Michelsen, S. Michelsen, M. Salewski, and E. L. Tsakadze, AIP Conf. Proc. 988, 118 (2008).

${ }^{19}$ M. Salewski, F. Meo, H. Bindslev, V. Furtula, S. B. Korsholm, B. Lauritzen, F. Leipold, P. K. Michelsen, S. K. Nielsen, and E. Nonbøl, Rev. Sci. Instrum. 79, 10E729 (2008).

${ }^{20}$ R. J. Wylde, IEE Proc., Part H: Microwaves, Antennas Propag. 131, 258 (1984). 